\title{
Comparison of ${ }^{18} \mathrm{~F}$-sodium fluoride PET/CT, ${ }^{18} \mathrm{~F}$-fluorocholine PET/CT and diffusion- weighted MRI for the detection of bone metastases in recurrent prostate cancer: a cost-effectiveness analysis in France
}

\author{
Mathieu Gauthé ${ }^{1,2,3^{*}}$ (1), Kevin Zarca ${ }^{2}$, Cyrielle Aveline ${ }^{1}$, Frédéric Lecouvet ${ }^{4}$, Sona Balogova ${ }^{3}$, Olivier Cussenot ${ }^{5}$,
} Jean-Noël Talbot ${ }^{1}$ and Isabelle Durand-Zaleski ${ }^{2,6}$

\begin{abstract}
Background: The diagnostic performance of ${ }^{18} \mathrm{~F}$-sodium fluoride positron emission tomography/computed tomography (PET/CT) (NaF), ${ }^{18}$ F-fluorocholine PET/CT (FCH) and diffusion-weighted whole-body magnetic resonance imaging (DW-MRI) in detecting bone metastases in prostate cancer (PCa) patients with first biochemical recurrence $(B C R)$ has already been published, but their cost-effectiveness in this indication have never been compared.
\end{abstract}

Methods: We performed trial-based and model-based economic evaluations. In the trial, PCa patients with first BCR after previous definitive treatment were prospectively included. Imaging readings were performed both on-site by local specialists and centrally by experts. The economic evaluation extrapolated the diagnostic performances of the imaging techniques using a combination of a decision tree and Markov model based on the natural history of PCa. The health states were non-metastatic and metastatic BCR, non-metastatic and metastatic castration-resistant prostate cancer and death. The state-transition probabilities and utilities associated with each health state were derived from the literature. Real costs were extracted from the National Cost Study of hospital costs and the social health insurance cost schedule.

Results: There was no significant difference in diagnostic performance among the 3 imaging modalities in detecting bone metastases. FCH was the most cost-effective imaging modality above a threshold incremental costeffectiveness ratio of 3000€/QALY when imaging was interpreted by local specialists and 9000€/QALY when imaging was interpreted by experts.

Conclusions: FCH had a better incremental effect on QALY, independent of imaging reading and should be preferred for detecting bone metastases in patients with biochemical recurrence of prostate cancer.

Trial registration: NCT01501630. Registered 29 December 2011.

Keywords: Prostate cancer, Medico-economic, Bone metastases

\footnotetext{
* Correspondence: mathieugauthe@yahoo.fr

This work was conducted in the AP-HP Health Economics Research Unit, INSERM UMR 1153, Paris, France.

${ }^{1}$ Nuclear Medicine, Hôpital Tenon, AP-HP, Sorbonne Université, 4 rue de la Chine, 75020 Paris, France

${ }^{2}$ AP-HP Health Economics Research Unit, INSERM UMR 1153, Paris, France

Full list of author information is available at the end of the article
}

(c) The Author(s). 2020 Open Access This article is distributed under the terms of the Creative Commons Attribution 4.0 International License (http://creativecommons.org/licenses/by/4.0/), which permits unrestricted use, distribution, and reproduction in any medium, provided you give appropriate credit to the original author(s) and the source, provide a link to the Creative Commons license, and indicate if changes were made. The Creative Commons Public Domain Dedication waiver (http://creativecommons.org/publicdomain/zero/1.0/) applies to the data made available in this article, unless otherwise stated. 


\section{Background}

Prostate cancer (PCa) is the second most prevalent cancer in men worldwide, accounting for approximately $15 \%$ of all diagnosed cancers [1], presenting an annual incidence of 31.1 per 100,000 cases and being the 3rd cause of death by cancer in men behind lung and colorectal cancers [1].

Bone is the most frequent metastatic site of $\mathrm{PCa}$ and is the only metastatic site in approximately $62 \%$ of cases [2]. The prevalence of bone metastasis in $\mathrm{PCa}$ is $3 \%$ at diagnosis (for all stages), and its estimated cumulative incidence is $16.6 \% 5$ years after PCa diagnosis [3]. The discovery of bone metastases marks a turning point in the history of the disease, especially in terms of treatment strategy and prognosis, and patients with bone metastases have a worse prognosis [3-5].

${ }^{18} \mathrm{~F}$-sodium fluoride positron emission tomography/ computed tomography (PET/CT) (NaF), ${ }^{18} \mathrm{~F}$-fluorocholine PET/CT (FCH) and diffusion-weighted whole-body magnetic resonance imaging (DW-MRI) are three novel imaging techniques that are effective for the detection of PCa bone metastases and their results may impact the management of patients [6]. Their diagnostic performances in detecting bone metastases in $\mathrm{PCa}$ patients with first biochemical recurrence (BCR) have already been published [7-9]. However, to the best of our knowledge, their cost-effectiveness in this indication has never been compared.

The objective of this study was to provide evidence for policy making at the national level by comparing the cost-effectiveness of the detection of bone metastasis in PCa patients with first BCR by means of these three imaging modalities.

\section{Methods}

The French multicentre study "FLUPROSTIC" (NCT01501630) was a prospective integrated clinical and economic national multicentre study comparing $\mathrm{NaF}, \mathrm{FCH}$ and DW-MRI in detecting bone metastasis in $\mathrm{PCa}$ patients with first BCR. The medical objective was to compare the diagnostic performance and the impact on patient management of these three imaging modalities in this indication. It was completed by a cost effectiveness analysis using real treatment costs of trial patients. The FLUPROSTIC trial was conducted in accordance with the Declaration of Helsinki and approved by a national review board (IDRCB 2011-A01041-40). All patients provided written informed consent. The study design, inclusion/exclusion criteria, follow-up and standard of truth (SOT) for bone metastasis that were applied in this study are summarized in Additional file 1. The imaging protocols and imaging interpretation are detailed below. For the purpose of economic evaluation, we extrapolated the results of the trial using a statetransition model.

\section{Population}

All PCa patients included in the FLUPROSTIC trial presenting with first $\mathrm{BCR}$ after previous definitive treatment for localized $\mathrm{PCa}$, without ongoing androgen-deprivation therapy (ADT), were considered in this costeffectiveness analysis. BCR was diagnosed after surgery, radiotherapy or alternative local treatment options with curative intent according to current recommendations [10]: two consecutive rising PSA values above $0.2 \mathrm{ng} / \mathrm{ml}$ following radical prostatectomy or any PSA increase greater than or equal to $2 \mathrm{ng} / \mathrm{ml}$ higher than the PSA nadir value, regardless of the nadir value, for nonsurgical first-line definitive treatments (radiation therapy, brachytherapy, high-intensity focused ultrasound). Each patient was treated and followed up by his referring physician after the imaging workup according to standards of care (French Association Urology guidelines, which are similar to those of the European Association of Urology) [11, 12]. Patients for whom the SOT for bone metastasis was not feasible were excluded from the cost-effectiveness analysis because it was not possible to categorize them into a health state. Patients for whom the follow-up duration after the imaging workup was less than 1 year were also excluded from the medicoeconomic analysis, because it was not possible to evaluate their annual treatment costs.

\section{Imaging analysis}

The imaging protocols are detailed in Additional file 2.

\section{Pet/CT}

A local nuclear physician with more than 5 years of experience in $\mathrm{PET} / \mathrm{CT}$ reading prospectively read the PET/ CT examinations the same day as the acquisition, not blinded to the results of other imaging, and provided a report of his analysis to the local clinicians (on-site reading of imaging). Two board-certified nuclear medicine physicians with 10 years of experience in PET/CT reading (one for $\mathrm{NaF}$ and one for $\mathrm{FCH}$ ), blinded to the clinical and other imaging results, performed retrospective randomized readings independently of each other (central masked reading of imaging). All PET/CT examinations were reviewed in a three-panel mode, displaying CT-scan, FCH/NaF-scan and fusion images, using a dedicated workstation (Syngo.via, Siemens Healthcare).

For image quotation, the skeleton was parted into 8 regions: skull, thoracic cage, cervical, thoracic spine, lumbar spine, pelvis, humeri, and femurs. Any focal $\mathrm{FCH}$ or NaF uptake above the background in the bone not corresponding to a benign pathology on CT (around 
the joints, osteophytes, fractures, etc.) was reported as positive $[9,13]$.

\section{MRI}

A local oncoradiologist with more than 10 years of experience in MRI reading prospectively read the MRI examinations the same day as the acquisition, not blinded to the results of other imaging, and provided a report of his analysis to the local clinicians (on-site reading of imaging). A board-certified radiologist with more than 20 years of experience in bone marrow MRI imaging and cancer imaging, blinded to the clinical and other imaging results, performed retrospective randomized readings independently of the PET/CTs (central masked reading of imaging). All images were read on PACS workstations (Carestream Vue; Carestream Health).

The 8 anatomic regions of the skeleton were the same as those for PET/CTs. A focal bone metastasis was defined as a rounded focus larger than $5 \mathrm{~mm}$ with low signal intensity on T1-weighted images and for the evaluation of the whole whole-body MRI examination, of low signal intensity on T1, intermediate to high signal intensity on STIR, and high signal intensity on the high b-value DWI sequence. Diffuse bone metastasis was defined as low signal intensity of the bone marrow (lower than the signal intensity of disks and muscles) on T1, intermediate to high signal intensity on STIR, and high signal intensity on the high b-value DWI sequence $[14,15]$.

\section{Economic evaluation}

The economic evaluation was conducted from the perspective of the French health care system. The short follow-up duration in the trial did not fully capture the medical and economic consequences of choosing between imaging techniques.

\section{Model structure}

We constructed a combination of a decision tree and Markov model based on the natural history of $\mathrm{PCa}$ (Fig. 1).

The decision tree was established by using the diagnostic accuracies of NaF, FCH and DW-MRI according to the established SOT for bone metastases. Markov models can be used to extrapolate the outcomes of a clinical trial over a longer time period, particularly in diseases with events that have ongoing risk or acute events that may occur more than once over the lifetime of a patient. In a Markov model, the course of a disease is represented by "states": some chronic and some acute depending on the disease. The patient is always in one of a finite number of states of health referred to as Markov states. Everything that is important in the course of a disease is listed and the patient at each point in time has a given probability to either remain in that state or to transition to another. The probabilities of transitioning or not transitioning are calculated by increments of time, referred to as Markov cycles. During each cycle, the patient may make a transition from one state to another but not more, so the maximum duration of a cycle is medically defined. Moreover, based on the medical aspects of a disease, some transitions are possible, and others are not, e.g., the transition from "progression free" to "progressed" is possible, but the opposite is not. The probability of making a transition from one state to another during a single cycle is called a transition probability. The Markov model is defined by the probability distribution among the states and the probabilities of the transitions allowed. The "death" state is the absorbing state. For the purpose of economic analyses, each state is characterized by a cost and a weight that reflects the quality of life of a patient in that state [16].

Two analyses were thus conducted: one using the onsite reading accuracies of imaging and a second using

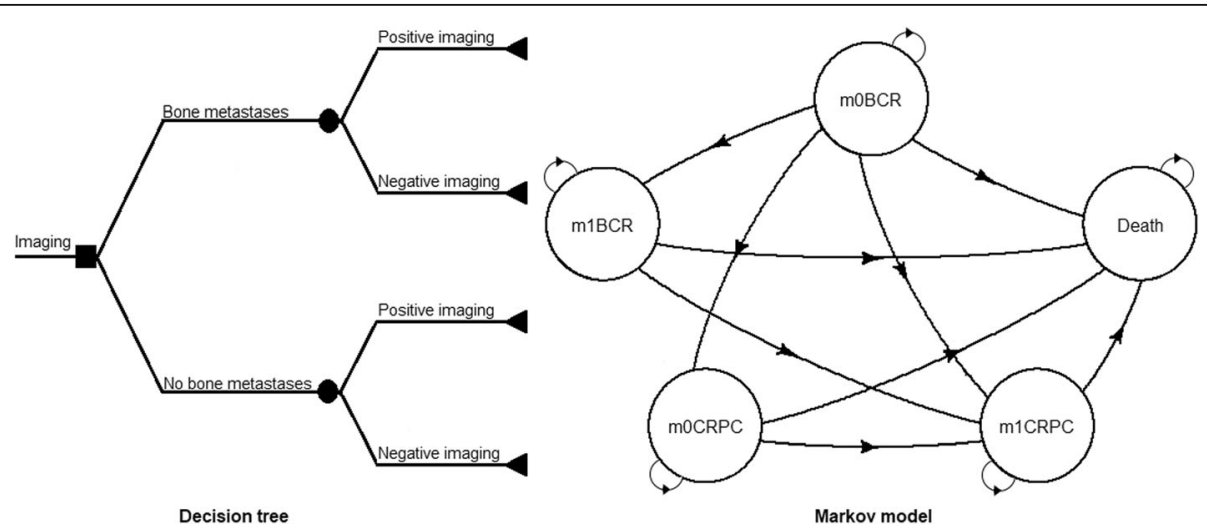

Fig. 1 Decision tree combined with the Markov model used for evaluating costs and health-related outcomes. BCR = patients with first biochemical recurrence of prostate cancer; CRPC: patient with castration-resistant prostate cancer; $\mathrm{m0}$ and $\mathrm{m} 1$ : patient with and without bone metastases, respectively 
the masked reading accuracies of imaging. To model the long-term health economics outcomes, we simulated a cohort of 10,000 PCa patients with first BCR at presentation identical to the trial patients, starting at the age of 70 years, which was the median age of the patients with first BCR in the FLUPROSTIC cohort, over a lifetime horizon. The Markov model had one-year cycles and consisted of 5 states: non-metastatic to bone $(\mathrm{m} 0) \mathrm{BCR}$, metastatic to bone $(\mathrm{m} 1) \mathrm{BCR}, \mathrm{m} 0$ castration-resistant prostate cancer (CRPC), m1-CRPC and death (Fig. 1). Patients with a correct diagnosis of bone metastases (true positives and true negatives of imaging) started in the $\mathrm{m} 0-\mathrm{BCR}$ or $\mathrm{m} 1-\mathrm{BCR}$ states depending on their bone metastatic status. Misdiagnosed nonmetastatic to bone patients (false positives of imaging) started in the $\mathrm{m} 0$ BCR state with the costs and quality of life of m1-BCR state for one cycle only. Misdiagnosed metastatic to bone patients (false negatives of imaging) entered the model in a tunnel state of one-cycle duration, and the diagnosis was corrected after 1 cycle as we noted in our series that the diagnosis of bone metastases was corrected on average during this time frame. Then, those patients entered m1-BCR, m1-CRPC or death states.
The state-transition probabilities associated with each health state were derived from the literature (Table 1) [2, 17-24]. A time-dependency was implemented for age, meaning that patients were aged 1 year each cycle.

\section{Costs}

Real treatment costs (updated to 2016 Euros $(€)$ ) were calculated for each patient included in the trial for the year following his inclusion. The costs of inpatients and outpatients were established based on activity logs completed by hospital practitioners, by considering the diagnosis-related group, healthcare common procedure coding system and ambulatory payment classification codes. The costs of out-of-hospital care (biology, imaging and medical consultations) were based on activity logs completed by the referring physician. Inpatients, outpatients and ambulatory care costs were calculated according to the data of the French National Study of health costs [25]. The costs of pharmaceuticals were based on prices listed by the French Public Welfare Agency [26]. The treatment costs that were used are summarized in Table 2. The detailed costs are provided in Additional file 3 . We assumed that salvage radiation

Table 1 Health state annual transition probabilities and utilities used in the model

\begin{tabular}{|c|c|c|}
\hline & Transition probabilities ( $95 \%$ Confidence Interval) & References \\
\hline \multicolumn{3}{|l|}{$\mathrm{m} 0 \mathrm{BCR}$} \\
\hline$\rightarrow \mathrm{m} 1 \mathrm{BCR}$ & $0.0288(0.0279-0.0297)$ & Hernandez 2018 [17] \\
\hline$\rightarrow \mathrm{m} 0 \mathrm{CRPC}$ & $0.0279(0.0249-0.0308)$ & Hirst 2012 [18] \\
\hline$\rightarrow \mathrm{m} 1 \mathrm{CRPC}$ & Combination $\mathrm{P}(\mathrm{m} 1 \mathrm{BCR}+\mathrm{m0} \mathrm{CRPC})$ & \\
\hline$\rightarrow \mathrm{m0}$ BCR Death & French male mortality in 2017 (time-dependant) & Ined.fr [19] \\
\hline On m0 BCR & 1 - others $P_{m 0} B C R$ & \\
\hline \multicolumn{3}{|l|}{ mo CRPC } \\
\hline$\rightarrow \mathrm{m} 1 \mathrm{CRPC}$ & $0.1520(0.1080-0.1940)$ & Smith 2005 [20] \\
\hline$\rightarrow \mathrm{m0}$ CRPC Death & Combination $\mathrm{P}(0.0413$ + French male mortality in 2017) & Hirst 2012, Ined.fr $[18,19]$ \\
\hline On m0 CRPC & 1 - others $P_{\mathrm{mo}}$ CRPC & \\
\hline \multicolumn{3}{|l|}{$\mathrm{m} 1 \mathrm{BCR}$} \\
\hline$\rightarrow \mathrm{m} 1 \mathrm{CRPC}$ & $0.2055(0.1813-0.2251)$ & James 2015 [2] \\
\hline$\rightarrow \mathrm{m} 1 \mathrm{BCR}$ Death & Combination P(0.1306 + French male mortality in 2017) & James 2015, Ined.fr $[2,20]$ \\
\hline On m1 BCR & 1 - others $P_{m 1} B C R$ & \\
\hline \multicolumn{3}{|l|}{ m1 CRPC } \\
\hline$\rightarrow \mathrm{m} 1 \mathrm{CRPC}$ Death & Combination P(0.2933 + French male mortality in 2017) & Fizazi 2011 [21] \\
\hline \multirow[t]{2}{*}{ On m1 CRPC } & 1 - others $P_{\mathrm{m} 1 \mathrm{CRPC}}$ & \\
\hline & Utilities (Standard Deviation) & References \\
\hline $\mathrm{m} 0 \mathrm{BCR}$ & $0.89(0.14)$ & Torvinen 2013 [22] \\
\hline mo CRPC & $0.86(0.17)$ & Saad 2018 [23] \\
\hline $\mathrm{m} 1 \mathrm{BCR}$ & $0.74(0.27)$ & Torvinen 2013 [22] \\
\hline m1 CRPC & $0.83(0.13)$ & Lloyd 2015 [24] \\
\hline Death & 0 & \\
\hline
\end{tabular}


Table 2 Annual costs in Euros for non-metastatic and metastatic to bone prostate cancer patients with biochemical recurrence

\begin{tabular}{lll}
\hline Cost item & Patients without bone metastases $(n=48)$ & Patients with bone metastases $(n=7)$ \\
\hline Androgen deprivation therapy & $523(364-695)$ & $691(244-1165)$ \\
Hospitalisation costs & & $3706(491-8138)$ \\
$\quad$ First year (with salvage radiation therapy) & $2601(1376-4381)$ & $3706(631-7973)$ \\
$\quad$ Other years & $921(431-1510)$ & $4816(1615-9234)$ \\
Total costs & & $4815(1689-9308)$ \\
$\quad$ First year & $3524(2277-5246)$ & $484(1354-2434)$
\end{tabular}

Mean annual treatment/management costs with $95 \%$ confidence interval. Total costs included hospitalization costs, monitoring costs (office visit, biology and imaging) and drugs costs

therapy of the prostatic lodge/pelvis was only performed once during the first year in eligible patients. Thus, its cost was considered only in the first cycle.

The annual management costs of $\mathrm{m} 0$ and $\mathrm{m} 1$-CRPC patients were calculated in the same way based on a series of $\mathrm{m} 0$ and $\mathrm{m} 1$-CRPC patients who were included in the FLUPROSTIC trial. Thus, the mean total annual management costs for CRPC patients which were used to run our model were $5717 €(95 \%$ CI: $1634-11,869)$ for m0-CRPC patients and 12,346€ (95\%CI: 3109-27,740) for m1-CRPC patients, based on a series of 15 patients including 4 patients with metastases. Misdiagnosed $\mathrm{m} 0$ BCR patients (false positive results of imaging) were attributed the costs of $\mathrm{m} 0-\mathrm{BCR}$ patients without counting the first-year salvage radiation therapy, and misdiagnosed $\mathrm{m} 1-\mathrm{BCR}$ patients (false negative results of imaging) were attributed the costs of $\mathrm{m} 0-\mathrm{BCR}$ patients for 1 year.

The annual production costs of each imaging modality per scan performed in the study hospital were calculated by adding the cost of the working time of each member of the staff involved in the care, the micro-costing and the depreciation of the PET/CT or MRI device (based on the buying cost, time to depreciate, annual maintenance and number of scans per year). The general costs of the medical centre were not considered for the calculation of the production costs as we assumed that they are the same for each imaging modality when performed in the same centre. The detailed production costs of each imaging modality are presented in Additional file 4.

A discount rate of $4 \%$ per annum was applied according to the current French recommendation for medicoeconomic studies [27].

\section{Utilities}

The utilities associated with each health state were derived from the literature (Table 1) [2, 17-24]. Misdiagnosed $\mathrm{m} 0-\mathrm{BCR}$ patients (false positive results of imaging) were attributed the utility score of $\mathrm{m} 1-\mathrm{BCR}$ patients and misdiagnosed $\mathrm{m} 1-\mathrm{BCR}$ patients (false negative results of imaging) entered the model in a tunnel state of one-cycle duration with the same utility as patients in the $\mathrm{m} 0$-BCR state.

\section{Sensitivity analyses}

Deterministic sensitivity analysis (DSA) was performed to evaluate the uncertainties surrounding relevant parameters within plausible ranges of $95 \%$ confidence intervals or standard deviations (according to the type of distribution), including the transition probability of developing bone metastasis, QALYs and treatment costs for $\mathrm{m} 0$ and $\mathrm{m} 1-\mathrm{BCR}$ patients.

A probabilistic sensitivity analysis was conducted to assess the effects of all parameter uncertainties, with a total of 1000 iterations of a Monte Carlo simulation. We used a binomial distribution for the transition probabilities of developing bone metastasis or resistance to castration and for QALYs, and a gamma distribution for treatment costs.

\section{Model validation}

We checked the internal validity of the Markov model by calculating the life expectancy of patients and comparing the results to French data [19].

\section{Statistical analysis}

The data were analysed using $\mathrm{R}$ software. The heemod package [28] was used to calculate the transition probabilities from annual rates, run the Markov model, calculate the incremental cost-effectiveness ratio (ICER) and perform deterministic and probabilistic sensitivity analyses. The patient-based diagnostic performances of the three imaging modalities were compared by using the Cochran Q test with McNemar chi-square as a post hoc test. The agreement between the on-site and central readings for bone metastases was assessed for the $3 \mathrm{im}$ aging modalities using Cohen's kappa coefficient.

The economic evaluation followed the CHEERS recommendations [29]. 


\section{Results}

\section{Base case: population and performance of imaging} modalities

Of the 59 patients with biochemical recurrence enrolled in the FLUPROSTIC trial, 4 were excluded because the SOT could not be determined. The data of 55 BCR patients prospectively included between December 2011 and August 2014 were thus analysed. At least one bone metastasis was found in 7/55 (12.7\%) patients (Table 3).
The mean duration of patient follow-up was 3 years (range: $1-7$ years).

There was no significant difference in diagnostic performance among the 3 imaging modalities in detecting bone metastases. The performances of each imaging modality in detecting bone metastases in BCR patients for on-site and central readings and the agreement between the two readings are presented in Table 4.

Table 3 Characteristics of included prostate cancer patients with biochemical recurrence

\begin{tabular}{|c|c|c|c|}
\hline Parameter & All patients & $\begin{array}{l}\text { Patients without } \\
\text { bone metastases } \\
\text { (mOBCR) }\end{array}$ & $\begin{array}{l}\text { Patients with bone } \\
\text { metastases } \\
\text { (m1BCR) }\end{array}$ \\
\hline $\mathrm{n}$ & 55 & 48 & 7 \\
\hline \multicolumn{4}{|l|}{ Median age in years [range] } \\
\hline At prostate cancer diagnosis & $65[46-78]$ & $65[46-78]$ & $66[55-76]$ \\
\hline At first biochemical recurrence & $71[50-87]$ & $71[50-86]$ & $72[56-87]$ \\
\hline \multicolumn{4}{|l|}{ Initial group according to d'Amico classification } \\
\hline Low risk & $7(13 \%)$ & 7 & 0 \\
\hline Intermediate risk & $23(42 \%)$ & 20 & 3 \\
\hline High risk & 19 (35\%) & 15 & 4 \\
\hline Unknown & $6(10 \%)$ & 6 & 0 \\
\hline \multicolumn{4}{|l|}{ Initial Gleason score } \\
\hline$\leq 6$ & $13(24 \%)$ & 13 & 0 \\
\hline 7 & $30(55 \%)$ & 25 & 5 \\
\hline$\geq 8$ & $8(15 \%)$ & 8 & 0 \\
\hline Unknown & $4(6 \%)$ & 2 & 2 \\
\hline \multicolumn{4}{|l|}{ Initial International Society of Urological Pathologists (ISUP) 2014 grade group } \\
\hline 1 & $13(24 \%)$ & 13 & 0 \\
\hline 2 & 17 (30\%) & 13 & 4 \\
\hline 3 & $10(18 \%)$ & 10 & 0 \\
\hline 4 & $7(13 \%)$ & 7 & 0 \\
\hline 5 & $1(2 \%)$ & 1 & 0 \\
\hline Unknown & $7(13 \%)$ & 4 & 3 \\
\hline \multicolumn{4}{|l|}{ First line treatment } \\
\hline Surgery (prostatectomy \pm lymph node dissection) & $29(53 \%)$ & 28 & 1 \\
\hline Definitive radiation therapy \pm ADT & 19 (35\%) & 14 & 5 \\
\hline Other local treatment options* & $7(12 \%)$ & 6 & 1 \\
\hline Median time to biochemical recurrence in months [range] & 89 [4-228] & $92[4-228]$ & $87[6-149]$ \\
\hline Median PSA serum value at BCR imaging workup ng/ml [range] & $4.7[0.2-137]$ & $4.1[0.2-52]$ & $16.5[1.0-137]$ \\
\hline \multicolumn{4}{|l|}{ Management of biochemical recurrence after imaging workup } \\
\hline Salvage radiation therapy (prostatic lodge \pm pelvic lymph nodes) \pm ADT & $8(15 \%)$ & 8 & 0 \\
\hline ADT & $27(49 \%)$ & 22 & 5 \\
\hline Surveillance & $9(16 \%)$ & 9 & 0 \\
\hline Other treatment option** & $11(20 \%)$ & 9 & 2 \\
\hline
\end{tabular}

ADT androgen deprivation therapy; *: 4 brachytherapy and 3 high-intensity focused ultrasound (HIFU); **: 1 pelvic lymph node dissection, 7 HIFU, 1 cryoablation, 1 radiation therapy of an isolated bone metastasis and 1 surgery of 2 lung metastasis 
Table 4 Performances of imaging in detecting bone metastases of prostate cancer patients with first biochemical recurrence (patient-base analysis)

\begin{tabular}{clllll}
\hline & Se & Sp & PPV & NPV & Accuracy \\
\hline $\begin{array}{c}\text { NaF PET/CT } \\
\text { On-site }\end{array}$ & $71 \%(5 / 7)$ & $92 \%(44 / 48)$ & $56 \%(5 / 9)$ & $96 \%(44 / 46)$ & $89 \%(49 / 55)$ \\
Central & $86 \%(6 / 7)$ & $94 \%(45 / 48)$ & $67 \%(6 / 9)$ & $98 \%(45 / 46)$ & $93 \%(51 / 55)$ \\
FCH PET/CT & & & & & \\
On-site & $43 \%(3 / 7)$ & $100 \%(48 / 48)$ & $100 \%(3 / 3)$ & $92 \%(48 / 52)$ & $93 \%(51 / 55)$ \\
Central & $57 \%(4 / 7)$ & $98 \%(47 / 48)$ & $80 \%(4 / 5)$ & $94 \%(47 / 50)$ & $93 \%(51 / 55)$ \\
DW-MRI & & & & & 0.86 \\
On-site & $57 \%(4 / 7)$ & $83 \%(40 / 48)$ & $33 \%(4 / 12)$ & $93 \%(40 / 43)$ & $80 \%(40 / 55)$ \\
Central & $43 \%(3 / 7)$ & $94 \%(45 / 48)$ & $60 \%(3 / 5)$ & $90 \%(45 / 50)$ & $87 \%(48 / 55)$ \\
\hline
\end{tabular}

Se sensitivity, $S p$ specificity, $P P V$ positive predictive value, NPV negative predictive value

$\mathrm{NaF}{ }^{18} \mathrm{~F}$-sodium fluoride, $\mathrm{FCH}{ }^{18} \mathrm{~F}$-fluorocholine, $\mathrm{DW}$-MRI diffusion-weighted whole-body magnetic resonance imaging

$K$ Cohen's kappa coefficient

FCH was a more cost-effective imaging modality in both analyses. The economic evaluation using the onsite imaging reading accuracies showed an extended dominance of FCH compared to NaF (Table 5) (Fig. 2). The ICER was $993 €$ per QALY gained, with an incremental cost of $481 €$ and an average difference of 0.48 QALYs. For the economic evaluation using the central imaging reading accuracies, $\mathrm{NaF}$ was strictly dominated (Table 5) (Fig. 2). The ICER was 5055€ per QALY gained, with an incremental cost of $666 €$ and an average difference of 0.14 QALYs.

\section{Sensitivity analyses}

The DSA showed that the uncertainty around the treatment costs of $\mathrm{m} 1-\mathrm{BCR}$ patients led in all cases to the largest variation in the ICER. These variations ranged from $730 €$ to $1547 €$ per QALY gained on the analyses using on-site imaging reading accuracies and from $4695 €$ to $5941 €$ per QALY gained on the analyses using central imaging reading accuracies (Fig. 3). All other tested parameters seemed to have minimal to moderate impact on the cost-effectiveness results (Fig. 3).
The probabilistic sensitivity analysis confirmed that FCH was the most cost-effective imaging modality in both analyses. It also confirmed the extended dominance of $\mathrm{FCH}$ compared to $\mathrm{NaF}$ when using on-site reading accuracies of imaging and that $\mathrm{NaF}$ was dominated when using the central imaging reading accuracies. The average incremental cost was $480 €$, the average difference was 0.47 QALYs and the average ICER was $1028 €$ for on-site imaging reading accuracy analyses and 649€, 0.14 and $5092 €$ respectively for central imaging reading accuracy analyses (Figs. 4 and 5). As shown in Fig. 5, FCH is probably the most cost-effective above a threshold ICER of $3000 €$ when imaging is interpreted by local specialists and of $9000 €$ when imaging is interpreted by experts.

We estimated that the overall life expectancy of PCa patients with $\mathrm{BCR}$ at the age of 70 years was 6.7 years, ranging from 4.1 years for m1-BCR to 9.2 years for $\mathrm{m} 0-\mathrm{BCR}$, when running the model without considering imaging accuracies.

\section{Discussion}

To the best of our knowledge, no previous study has ever reported a direct prospective head-to-head cost-

Table 5 Efficiency frontier and summary of cost-effectiveness results

\begin{tabular}{llll}
\hline Efficiency frontier (on-site reading) & MRI with DW-MRI & NaF-PET/CT & FCH-PET/CT \\
\hline Life expectancy (years) & 5.50 & 5.78 & 6.11 \\
QALYs & 4.45 & 4.67 & 4.93 \\
Cost in Euros & 22,160 & 22,385 & 22,641 \\
ICER: Euros per QALY gained & & 1002 & MRI with DW-MRI \\
Efficiency frontier (central reading) & NaF-PET/CT & 593 \\
Life expectancy (years) & 5.87 & 5.87 & FCH-PET/CT \\
QALYs & 4.73 & 4.73 & 6.03 \\
Cost in Euros & 22,481 & 22,063 & 4.87 \\
ICER: Euros per QALY gained & dominated & & 22,729 \\
\hline
\end{tabular}

ICER Incremental cost-effectiveness ration; QALYs Quality-adjusted life expectancy 

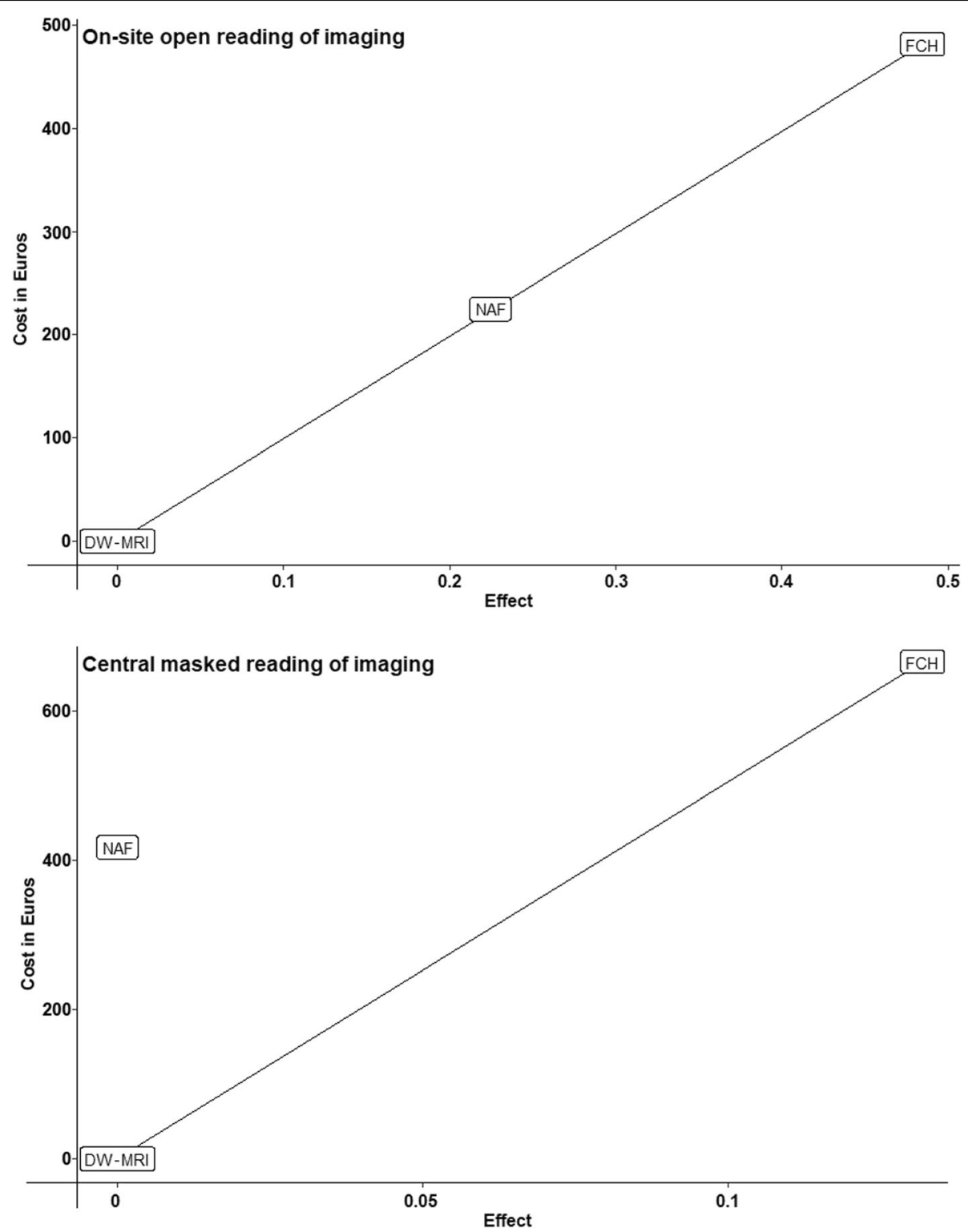

Fig. 2 Incremental cost in Euros and effect of imaging strategies on the cost-effectiveness plane. NaF $={ }^{18} \mathrm{~F}$-sodium fluoride $\mathrm{PET} / \mathrm{CT} ; \mathrm{FCH}={ }^{18} \mathrm{~F}-$ fluorocholine PET/CT; DW-MRI = diffusion-weighted whole-body magnetic resonance imaging

effectiveness comparison of $\mathrm{NaF}, \mathrm{FCH}$ and DW-MRI in the detection of bone spread in a homogenous group of PCa patients with first BCR.

\section{Imaging performances}

The diagnostic performances of $\mathrm{NaF}, \mathrm{FCH}$ and DW-MRI in this setting have already been published [7, 9, 30, 31]. The accuracy of $\mathrm{NaF}$ in detecting bone metastases was found to be significantly better than that of DW-MRI in a recent prospective comparison of $68 \mathrm{PCa}$ patients with BCR [7]. Langsteger et al. observed a better lesion-based specificity of FCH compared to that of $\mathrm{NaF}$ ( $96 \%$ vs $91 \%$, $p=0.033)$ for an equivalent sensitivity of $89 \%$ in the context of BCR [9]. In 2014, a meta-analysis compared the pooled performances of MRI (all variants, including DWMRI) and choline PET/CT (pooling results of FCH and ${ }^{11} \mathrm{C}$-choline) in the diagnosis of bone metastases in $\mathrm{PCa}$ patients [30]. Overall, a non-significantly lower sensitivity was found for choline PET/CT compared to MRI, with pooled sensitivities of 87 and $95 \%$, and without a difference in specificity values of 97 and $96 \%$ respectively. In 2016, Barchetti et al. performed FCH and DW-MRI in $152 \mathrm{PCa}$ patients with BCR [31]. They considered the FCH results as the SOT. DW-MRI had a detection rate of 99\% in patients presenting with bone spread on $\mathrm{FCH}$ [31]. In the present study, we found that the diagnostic 


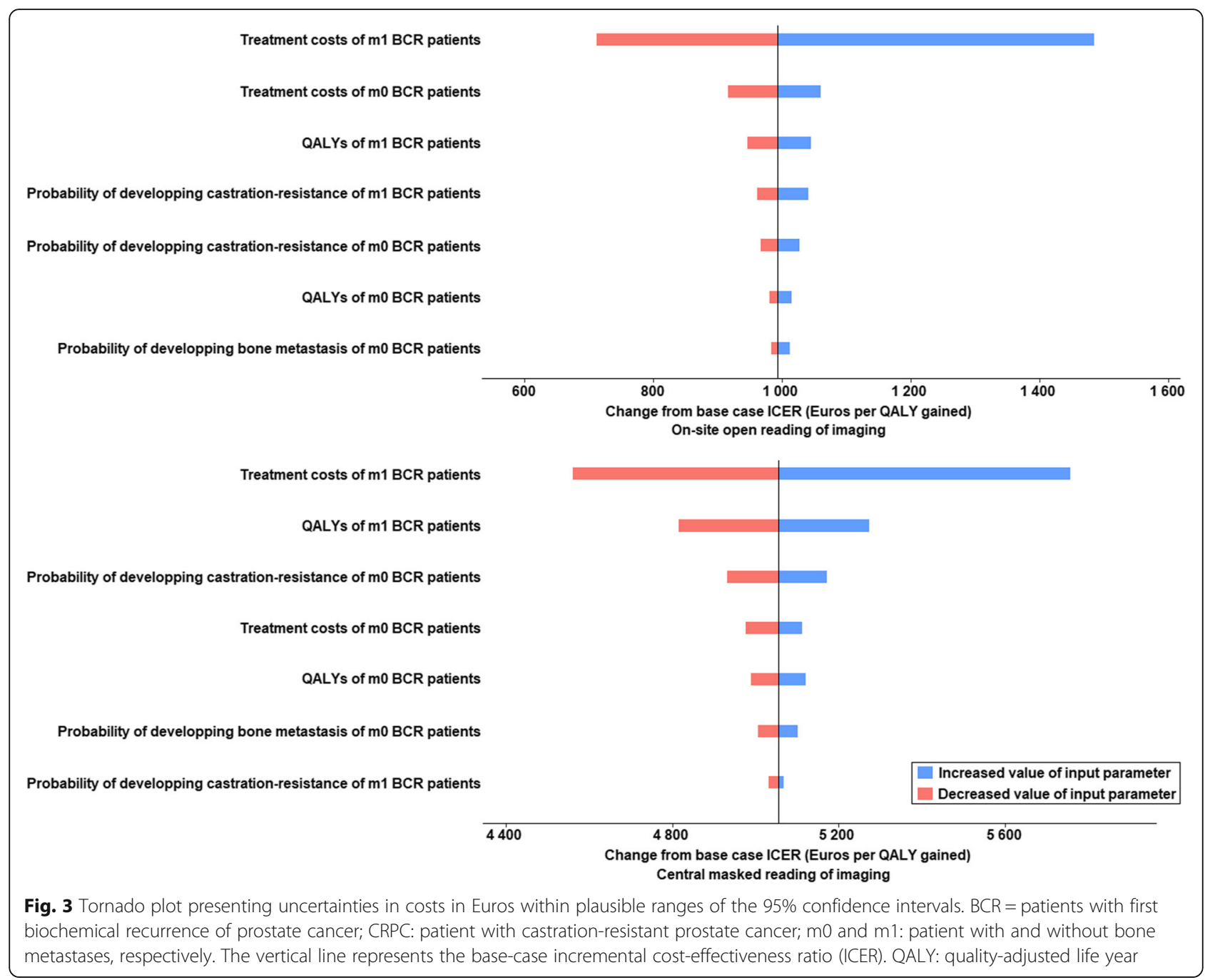

performances of the 3 imaging modalities in detecting bone metastases in PCa patients with BCR were concordant with the reported values of other published studies. Thus, we assumed that our procedures did not favour any imaging strategy in the medico-economic analysis.

\section{Medico-economic analysis}

In our study, $\mathrm{FCH}$ was always the most cost-effective imaging modality for staging patients with BCR, considering either the on-site reading by local specialists or the central reading by the experts of each imaging modality. $\mathrm{PET} / \mathrm{CT}$ is often criticized for its higher cost compared to other imaging modalities that can be prescribed to explore PCa. From the point of view of an imaging centre, production costs are higher for the PET/CTs than for MRI (302€ for $\mathrm{NaF}$ and $881 €$ for FCH versus $112 €$ for DW-MRI), but these differences are only because of the radiotracers' costs. Of note, no contrast agent was used for MRI in our study, which decreased MRI production costs as gadolinium is frequently injected into patients in routine practice for complementary sequences to DW-MRI. From the point of view of the French healthcare system, the reimbursed amounts for the PET/CTs, which are currently the same regardless of the radiotracer, are also approximately four times higher than those for MRI (Additional file 3). However, as shown in Table 5 and illustrated in Fig. 4, imaging costs have nearly no impact on overall patient care costs, as a difference of only $481 €$ and $666 €$ exists between the cheapest strategy (DW-MRI) and the most expensive strategy $(\mathrm{FCH})$ when using on-site and central imaging performances respectively. On the other hand, $\mathrm{FCH}$ had higher QALY (0.48 and 0.14 with on-site and central imaging performances respectively) than DW-MRI.

\section{Limitations}

The main factor shared by all studies addressing metastatic bone spread is the lack of histological evidence for 

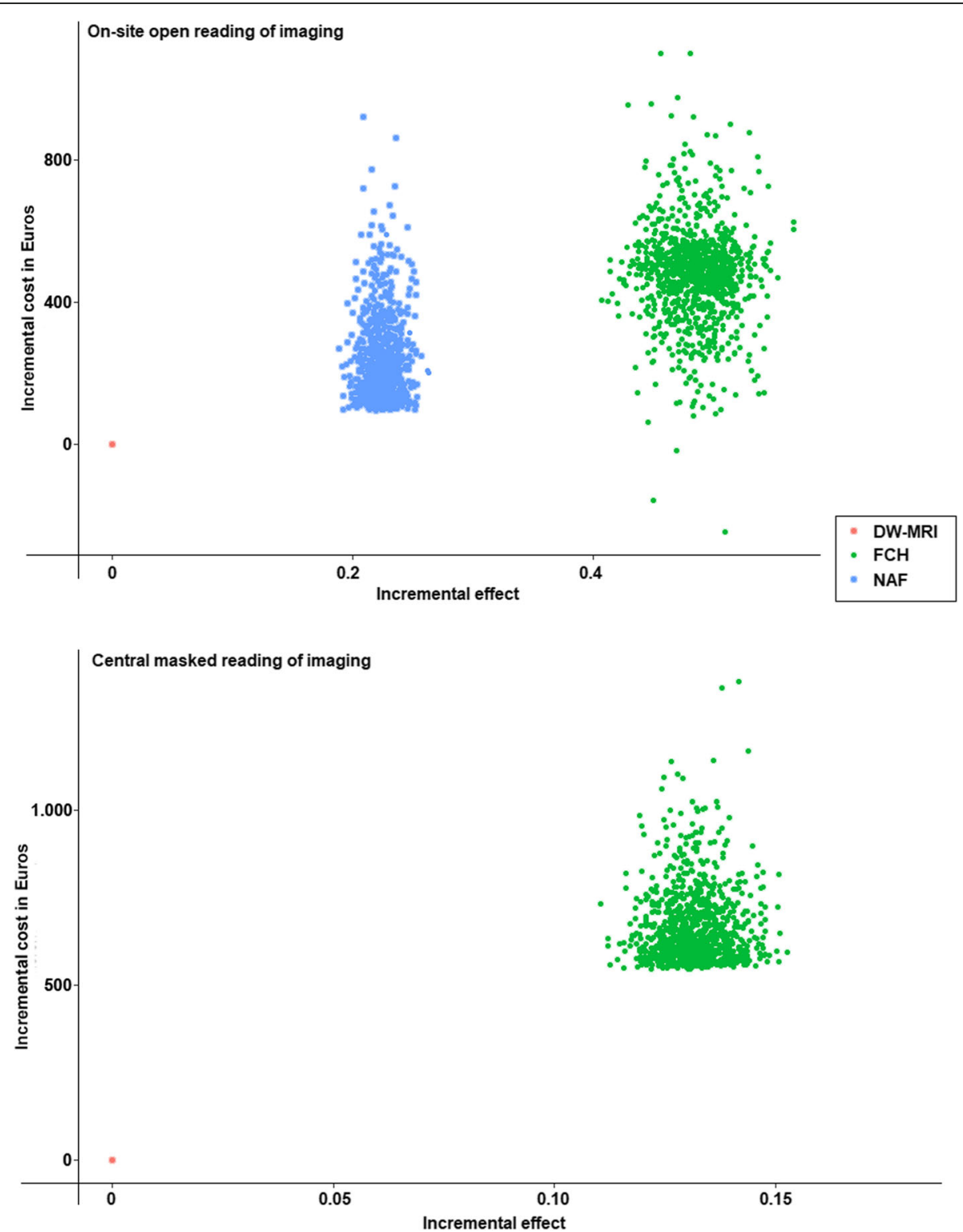

Fig. 4 Scatter plot showing the uncertainty of the incremental cost-effectiveness ratio in Euros for each imaging modality. ICER =incremental cost-effectiveness ratio; $\mathrm{NaF}={ }^{18} \mathrm{~F}$-sodium fluoride PET/CT; FCH $={ }^{18} \mathrm{~F}$-fluorocholine PET/CT; DW-MRI = diffusion-weighted whole-body magnetic resonance imaging

most metastases, which were mainly characterized on the basis of follow-up data. However, this limitation applied equally to the 3 imaging modalities and it is assumed that it would not have favoured one of them.

The second major limitation of this work was the relatively limited number of included patients due to logistical difficulties in prospectively completing the entire imaging workup in the early 2010s. However, our study was the largest homogenous prospective head-to-head cost-effectiveness comparison of these 3 imaging modalities ever reported. The use of a Markov model of the disease was thus essential to simulate the outcomes of PCa patients with BCR in a larger number of patients on a lifetime horizon.

In this study we did not find significant differences among the 3 imaging modalities by using an analysis that summarized the performance of each imaging modality in detecting bone marrow involvement in a perpatient approach. The results could have been different by using a lesion-based analysis. However, we assume that this per-patient approach did not alter the impact on determining patient management or treatment costs, 


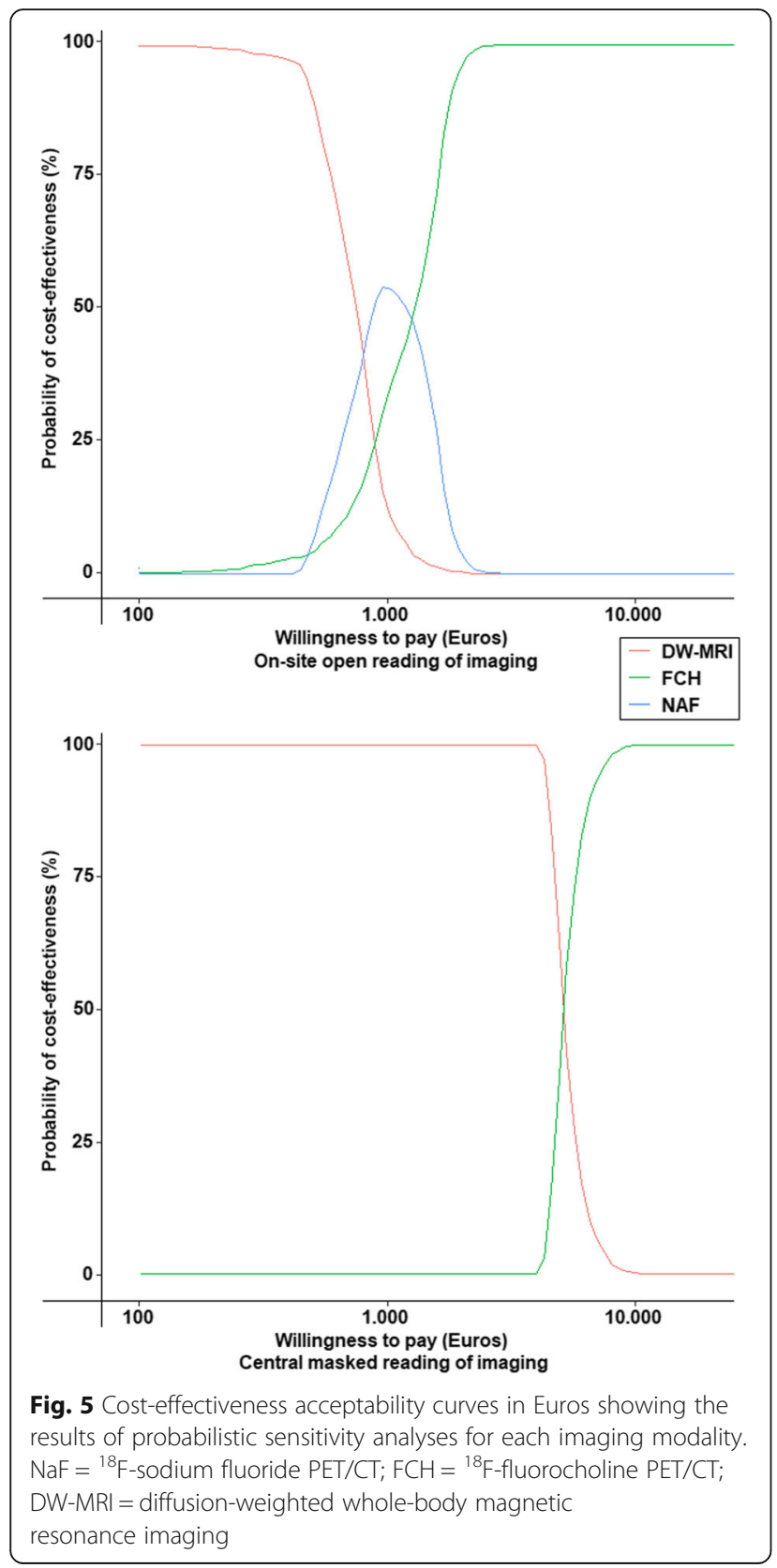

as in the real world therapeutic decision-making is based on a holistic approach of the patients' disease (patientbased) and not on a lesion per lesion approach (lesionbased).

We found better interobserver agreement between onsite and masked readings for the PET/CTs than for DWMRI. The low reproducibility of the DW-MRI readings may be explained by a lack of standardization of the analysis of this modality, which was recently prompted [32]. Indeed, the definition of bone, node and visceral metastases was settled by the time the study protocol was tailored, based on the available literature at that time, well before recent efforts for harmonization in image acquisition and reporting (MET-RADS criteria) [32]. Again, these differences in the reading agreement of imaging between on-site local specialists and central experts had almost no impact on the total cost of patient care.

The transition probabilities were extracted from the literature and are sometimes based on 10-year-old studies, whereas new therapeutics, such as secondgeneration anti-androgens such abiraterone acetate or enzalutamide, are now routinely prescribed to patients and may increase survival. Our model predicted an overall life expectancy of 6.7 years for PCa patients with BCR at the age of 70 years when running the simulation without considering the performance of imaging. This result was consistent with the average life expectancy of the French population of 79.4 for men in 2018 [19] and validated the model.

This study included patients with first BCR after previous definitive treatment for localized PCa. Some of them were treated with salvage high intensity focalized ultrasound (HIFU) after the imaging workup, through this alternative was not recommended at the time of patient inclusion in the FLUPROSTIC study. However, as HIFU is currently suggested in the EAU guidelines for the treatment of relapse for radiation-recurrent PCa [10], and as we used real treatment costs for the costeffectiveness study, we assume our results may be extended to the current context of recurrent PCa. Furthermore, as the health-state costs are the same for the 3 imaging modalities, none were penalized by this point.

We chose to evaluate the medico-economic impact of imaging by considering only their performance in detecting bone metastases, while PCa patient management may be based on the detection of lymph node metastases, especially for oligometastatic patients. Performing a medico-economic analysis regarding the performance of imaging in detecting lymph node metastases would have required the availability of relevant transition probabilities in the literature in this setting which is currently lacking for such data. Such analysis could not have been performed for NaF, which only explores bone metabolism.

Finally, with the advent of PET/CT using ligands of prostate-specific membrane antigen (PSMA) as a radiotracer, the usefulness of such cost-effectiveness highlighting a metabolic radiotracer such as $\mathrm{FCH}$ for PCa might be questioned. However, we demonstrated that the imaging modality that was used did not impact the total cost of patient care but influenced QALYs (Fig. 4). Thus, the model we developed could be used to compare the cost-effectiveness of different imaging modalities, including PSMA ligands radiotracers. 


\section{Conclusion}

$\mathrm{NaF}, \mathrm{FCH}$ and DW-MRI showed high diagnostic performances in detecting bone spread in prostate cancer patients with biochemical recurrence.

The cost-effectiveness analyses showed that imaging had no impact on the total costs of patient care.

FCH had a better incremental effect on QALY, independent of imaging reading, and should be preferred for imaging the biochemical recurrence of prostate cancer.

\section{Supplementary information}

Supplementary information accompanies this paper at https://doi.org/10. 1186/s12880-020-00425-y.

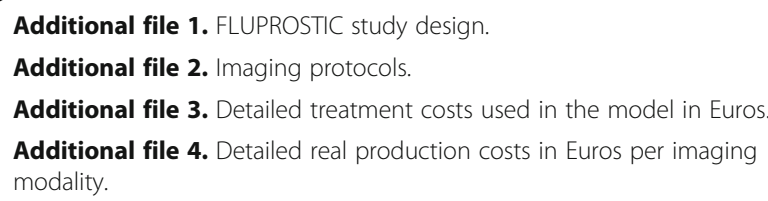

\section{Abbreviations}

ADT: Androgen-deprivation therapy; BCR: Biochemical recurrence; CRPC: Castration-resistant prostate cancer; DSA: Deterministic sensitivity analysis; DW-MRI: Diffusion-weighted whole-body magnetic resonance imaging; FCH: ${ }^{18}$ F-fluorocholine PET/CT; ICER: Incremental cost-effectiveness ratio; M0: Non-metastatic to bone; M1: Metastatic to bone; NaF: ${ }^{18}$ F-sodium fluoride; PCa: Prostate cancer; PET/CT: Positron emission tomography / computed tomography; PSA: Prostate-specific antigen; QALY: Qualityadjusted life-year; SOT: Standard of truth

\section{Acknowledgements}

The authors want to thank Mrs. Zakia Idir, AP-HP, and Prof. Eric Vicaut of URC Lariboisière for monitoring this study. They pay tribute Dr. Laure Michaud, Dr. Caroline Rousseau and all clinicians for referring their patients and acknowledge their confidence. Dr. Gauthé specially thanks Dr. Jérôme Frenkiel, Dr. Pierre Rufat, Dr. Odile Talvard and Ms. Jessica Weinstein for help in collecting cost data.

\section{Authors'contributions \\ MG: model conception; data collection and interpretation; manuscript writing. KZ: model conception; data and manuscript editing. CA: data revision and manuscript editing. FL: data revision and manuscript editing. SB: data revision and manuscript editing. OC: data revision and manuscript editing. JNT: study conception; data revision and manuscript editing. IDZ: study supervision; data revision and interpretation; manuscript editing. All authors have read and approved the manuscript as submitted.}

\section{Funding}

The French Ministry of Health performed a call for innovative project within the framework of a STIC (Support to Innovative and Costly Therapies). He selected and granted funds for the project "FLUPROSTIC" (STIC 2009 P090105) which was proposed by Pr JN Talbot (Médecine nucléaire, hospital Tenon, APHP). The funds were given to the Assistance Publique-Hôpitaux de Paris (APHP, French public hospital) to sponsor the study. Those are public funds.

\section{Availability of data and materials}

The datasets used and analysed during the current study are available from the corresponding author on reasonable request.

\section{Ethics approval and consent to participate}

The French multicentre study "FLUPROSTIC" (NCT01501630) was conducted in accordance with the Declaration of Helsinki. It was approved by the "Comité de protection des personnes Ile-de-France X" (IDRCB 2011-A010140), which is an official independent national ethical research committee certified by the French Ministry of Health.
All patients provided written informed consent.

Consent for publication

Not applicable.

\section{Competing interests}

The authors declare that they have no competing interests.

\section{Author details}

${ }^{1}$ Nuclear Medicine, Hôpital Tenon, AP-HP, Sorbonne Université, 4 rue de la Chine, 75020 Paris, France. ${ }^{2}$ AP-HP Health Economics Research Unit, INSERM UMR 1153, Paris, France. ${ }^{3}$ Nuclear Medicine, Faculty of Medicine, Comenius University and St. Elisabeth Oncology Institute, Bratislava, Slovakia.

${ }^{4}$ Radiology, Institut de Recherche Expérimentale Et Clinique, Cliniques

Universitaires Saint-Luc, Université Catholique de Louvain, Brussels, Belgium. ${ }^{5}$ Urology, Hôpital Tenon, AP-HP, Sorbonne Université, Paris, France.

${ }^{6}$ Department of Public Health, Hôpital Henri Mondor, AP-HP,Université Paris 12, Créteil, France.

Received: 8 February 2020 Accepted: 18 February 2020

Published online: 02 March 2020

\section{References}

1. Ferlay J, Soerjomataram I, Dikshit R, Eser S, Mathers C, Rebelo M, et al. Cancer incidence and mortality worldwide: sources, methods and major patterns in GLOBOCAN 2012. Int J Cancer. 2015:136:E359-86.

2. James ND, Spears MR, Clarke NW, Dearnaley DP, De Bono JS, Gale J, et al. Survival with newly diagnosed metastatic prostate cancer in the "docetaxel era": data from 917 patients in the control arm of the STAMPEDE trial (MRC PR08, CRUK/06/019). Eur Urol. 2015;67:1028-38.

3. Nørgaard $M$, Jensen $A \varnothing$, Jacobsen JB, Cetin K, Fryzek JP, Sørensen HT. Skeletal related events, bone metastasis and survival of prostate cancer: a population based cohort study in Denmark (1999 to 2007). J Urol. 2010;184: 162-7.

4. Singh D, Yi WS, Brasacchio RA, Muhs AG, Smudzin T, Williams JP, et al. Is there a favorable subset of patients with prostate cancer who develop oligometastases? Int J Radiat Oncol. 2004;58:3-10.

5. Sweeney CJ, Chen Y-H, Carducci M, Liu G, Jarrard DF, Eisenberger M, et al. Chemohormonal therapy in metastatic hormone-sensitive prostate cancer. N Engl J Med. 2015;373:737-46.

6. Gauthé M, Aveline C, Lecouvet F, Michaud L, Rousseau C, Tassart M, et al. Impact of sodium 18F-fluoride PET/CT, 18F-fluorocholine PET/CT and wholebody diffusion-weighted MRI on the management of patients with prostate cancer suspicious for metastasis: a prospective multicentre study. World J Urol. 2018. https://doi.org/10.1007/s00345-018-2547-5.

7. Zacho HD, Nielsen JB, Afshar-Oromieh A, Haberkorn U, de Souza N, De Paepe K, et al. Prospective comparison of 68Ga-PSMA PET/CT, 18F-sodium fluoride PET/CT and diffusion weighted-MRI at for the detection of bone metastases in biochemically recurrent prostate cancer. Eur J Nucl Med Mol Imaging. 2018;45:1884-97.

8. Gillebert Q, Huchet V, Rousseau C, Cochet A, Olivier P, Courbon F, et al. 18Ffluorocholine PET/CT in patients with occult biochemical recurrence of prostate cancer: detection rate, impact on management and adequacy of impact. A prospective multicentre study. PLOS ONE. 2018;13:e0191487.

9. Langsteger W, Balogova S, Huchet V, Beheshti M, Paycha F, Egrot C, et al. Fluorocholine (18F) and sodium fluoride (18F) PET/CT in the detection of prostate cancer: prospective comparison of diagnostic performance determined by masked reading. Q J Nucl Med Mol Imaging. 2011:55:44857.

10. Cornford P, Bellmunt J, Bolla M, Briers E, Santis MD, Gross T, et al. EAUESTRO-SIOG guidelines on prostate cancer. Part II: treatment of relapsing, metastatic, and castration-resistant prostate cancer. Eur Urol. 2017;71:63042.

11. Professionals S-O. EAU Guidelines: Oncology Guidelines. Uroweb https:// uroweb.org/individual-guidelines/oncology-guidelines/. Accessed 11 Dec 2019.

12. Recommandations de l'A.F.U. classées par année | Urofrance. https://www urofrance.org/outils-et-recommandations/recommandations/ recommandations-afu/classees-par-annee.html. Accessed 11 Dec 2019.

13. Beheshti M, Vali R, Waldenberger P, Fitz F, Nader M, Loidl W, et al. Detection of bone metastases in patients with prostate cancer by $18 \mathrm{~F}$ fluorocholine 
and $18 \mathrm{~F}$ fluoride PET-CT: a comparative study. Eur J Nucl Med Mol Imaging. 2008;35:1766-74

14. Vande Berg BC, Malghem J, Lecouvet FE, Maldague B. Classification and detection of bone marrow lesions with magnetic resonance imaging. Skelet Radiol. 1998;27:529-45.

15. Vanel D, Dromain C, Tardivon A. MRI of bone marrow disorders. Eur Radiol. 2000;10:224-9.

16. Sonnenberg FA, Beck JR. Markov models in medical decision making: a practical guide. Med Decis Mak. 1993;13:322-38.

17. Hernandez RK, Wade SW, Reich A, Pirolli M, Liede A, Lyman GH. Incidence of bone metastases in patients with solid tumors: analysis of oncology electronic medical records in the United States. BMC Cancer. 2018;18:44.

18. Hirst CJ, Cabrera C, Kirby M. Epidemiology of castration resistant prostate cancer: a longitudinal analysis using a UK primary care database. Cancer Epidemiol. 2012:36:e349-53.

19. Institut national de la statistique et des études économiques. Espérance de vie - Mortalité - Tableaux de l'économie française. https://www.insee.fr/fr/ statistiques/3676610?sommaire=3696937. Accessed 14 May 2019.

20. Smith MR, Kabbinavar F, Saad F, Hussain A, Gittelman MC, Bilhartz DL, et al. Natural history of rising serum prostate-specific antigen in men with castrate nonmetastatic prostate Cancer. J Clin Oncol. 2005;23:2918-25.

21. Fizazi K, Carducci M, Smith M, Damião R, Brown J, Karsh L, et al. Denosumab versus zoledronic acid for treatment of bone metastases in men with castration-resistant prostate cancer: a randomised, double-blind study. Lancet. 2011;377:813-22.

22. Torvinen S, Färkkilä N, Sintonen H, Saarto T, Roine RP, Taari K. Health-related quality of life in prostate cancer. Acta Oncol. 2013;52:1094-101.

23. Saad F, Cella D, Basch E, Hadaschik BA, Mainwaring PN, Oudard S, et al. Effect of apalutamide on health-related quality of life in patients with nonmetastatic castration-resistant prostate cancer: an analysis of the SPARTAN randomised, placebo-controlled, phase 3 trial. Lancet Oncol. 2018;19:140416.

24. Lloyd AJ, Kerr C, Penton J, Knerer G. Health-related quality of life and health Utilities in Metastatic Castrate-Resistant Prostate Cancer: a survey capturing experiences from a diverse sample of UK patients. Value Health. 2015;18: $1152-7$.

25. Agence technique de l'information sur l'hospitalisation. Référentiel national de coûts des prises en charge (ENC). https://www.scansante.fr/applications/ donnees-de-couts. Accessed 13 Feb 2019.

26. L'Assurance Maladie. Base des Médicaments et Informations Tarifaires. http://www.codage.ext.cnamts.fr/codif/bdm_it/index_presentation.php?.p_ site=AMELI. Accessed 13 Feb 2019.

27. Haute Autorité de Santé. Choix méthodologiques pour l'évaluation économique à la HAS. https://www.has-sante.fr/portail/jcms/__1499251/fr/ choix-methodologiques-pour--evaluation-economique-a-la-has. Accessed 21 Feb 2019.

28. Filipović-Pierucci A, Zarca K, Durand-Zaleski I. Markov Models for Health Economic Evaluations: The R Package heemod. ArXiv170203252 Stat. 2017. http://arxiv.org/abs/1702.03252. Accessed 26 Mar 2019.

29. Husereau D, Drummond M, Petrou S, Carswell C, Moher D, Greenberg D, et al. Consolidated health economic evaluation reporting standards (CHEERS) — explanation and elaboration: a report of the ISPOR health economic evaluation publication guidelines good reporting practices task force. Value Health. 2013;16:231-50.

30. Shen G, Deng H, Hu S, Jia Z. Comparison of choline-PET/CT, MRI, SPECT, and bone scintigraphy in the diagnosis of bone metastases in patients with prostate cancer: a meta-analysis. Skelet Radiol. 2014;43:1503-13.

31. Barchetti F, Stagnitti A, Megna V, Al Ansari N, Marini A, Musio D, et al. Unenhanced whole-body MRI versus PET-CT for the detection of prostate cancer metastases after primary treatment. Eur Rev Med Pharmacol Sci. 2016:20:3770-6.

32. Padhani AR, Lecouvet FE, Tunariu N, Koh D-M, De Keyzer F, Collins DJ, et al. METastasis reporting and data system for prostate Cancer: practical guidelines for acquisition, interpretation, and reporting of whole-body magnetic resonance imaging-based evaluations of multiorgan involvement in advanced prostate Cancer. Eur Urol. 2017;71:81-92.

\section{Publisher's Note}

Springer Nature remains neutral with regard to jurisdictional claims in published maps and institutional affiliations.

Ready to submit your research? Choose BMC and benefit from:

- fast, convenient online submission

- thorough peer review by experienced researchers in your field

- rapid publication on acceptance

- support for research data, including large and complex data types

- gold Open Access which fosters wider collaboration and increased citations

- maximum visibility for your research: over $100 \mathrm{M}$ website views per year

At BMC, research is always in progress.

Learn more biomedcentral.com/submissions 